\section{P389 CONGENITAL SYPHILIS IN A DEVELOPED COUNTRY - A CASE REPORT}

${ }^{1}$ Sheiniz Giva*, 'Wendy Ferguson, ${ }^{1}$ Roisin Egan, 'Abhidhamma Kaninde, ${ }^{2}$ Rebecca O'Sullivan, 'Joanne O'Gorman, 'Breda Hayes. 'Dept. of Neonatology, The Rotunda Hospital, Dublin, Ireland; ' ${ }^{2}$ niversity College Dublin, School of Medicine, Dublin, Ireland

\subsection{6/archdischild-2019-epa.735}

Background Congenital syphilis (CS) is an infectious disease caused by the spirochete Treponema pallidumacquired by the foetus in utero. Transmission is transplacental and it may occur at any stage during pregnancy.

Clinical Case A male infant born in Ireland at 37 weeks to an Irish lady of traveller ethnicity who did not seek antenatal care. The infant was unwell at birth requiring ventilatory and inotropic support; he had copious nasal secretions. Birth weight of $2.06 \mathrm{Kg}\left(<2^{\text {nd }}\right.$ centile $)$ and head circumference of $31 \mathrm{~cm}\left(2^{\text {nd }}\right.$ centile $)$ demonstrated growth restriction. The mother's delivery serology revealed positive T. pallidum(T.pallidum IgG/IgM positive), T. pallidum particle agglutination assay (TPPA) positive, Rapid Plasma Reagin(RPR) positive (1:128) and $T$. pallidum IgM positive. The mother had suffered 'flulike' symptoms during the pregnancy and had pruritus in the palms and soles.

Newborn Investigations Serological testing for T. pallidumwas positive (TPPA 1:2480, RPR 1:8). CSF testing was also performed (RPR titre 1;1, TPPA 1:640).

Other investigations also revealed thrombocytopenia $\left(35,000 / \mathrm{mm}^{3}\right)$ and elevated Alkaline Phosphatase (384 U/L) and Aspartate aminotransferase (85 U/L).

Abdominal ultrasound confirmed hepatosplenomegaly and cranial ultrasound showed cysts in the caudothalamic grooves bilaterally consistent with congenital infection.

Long bones $\mathrm{x}$-ray demonstrated a thick periosteal reaction in the diaphysis and metaphysis of the upper and lower limb bones bilaterally and a 'sawtooth pattern' of metaphyseal abnormality in the right distal radius and ulna which is pathognomonic of CS.

Treatment The infant received IV benzylpenicillin as per guidelines. Regular follow up is scheduled with the Infectious Diseases Team and other relevant services.

Discussion In the 21st century, a worldwide re-emergence of syphilis infection and an increase in early infectious syphilis (EIS) numbers and congenitally infected cases have been reported, notably in developed countries. In 2017, there were 398 notifications of EIS in Ireland (8.4 per 100,000 population compared to 3.7 per 100,00 population in 2012) and $25 \%$ of females with EIS were pregnant at the time of diagnosis.

Although the incidence of EIS in pregnancy is low (0.6/ 1000 births), early detection and treatment reduces the risk of CS. The consequences of untreated infection, as described in this case can be significant.

Conclusion This case highlights the continuing importance of antenatal screening in a low incidence country to identify EIS in pregnancy.

To our knowledge this is the first case of symptomatic CS born to a mother of ethnic Irish origin in 2 decades.

\section{P390 \\ DIFFERENCES DETECTED DURING FOLLOW-UP EXAMINATION OF CHILDREN WITH BRONCHIAL ASTHMA GROUPED BY THEIR PARASITOLOGIC STATUS IN EARLY CHILDHOOD}

${ }^{1}$ Mariya Kuropatenko* ${ }^{2}$ Liudmila Zhelenina. 'FSBSI 'Institute of Experimental Medicine', Saint Petersburg, Russian Federation; ${ }^{2}$ St.Petersburg State Pediatric Medical University, Ministry of Healthcare of the Russian Federation, Saint Petersburg, Russian Federation

\subsection{6/archdischild-2019-epa.736}

Background Despite a large number of clinical trials devoted to the subject, there are currently several controversial ideas about the interdependence between allergic diseases and parasitic invasions. This study analyses the dependence between the frequency of clinical manifestations of allergy (bronchial asthma, pollinosis, allergic rhinitis, atopic dermatitis) and the rate of sustained remission in 18-year-old adolescents who had been regularly examined due to their bronchial asthma since early childhood.

The aim of this study was to compare catamnestic data collected about children with bronchial asthma with regard to the presence or absence of concomitant helminthes and protozoa invasions in childhood.

Materials and methods Follow-up data was collected about 90 adolescents who had been treated for mild to moderate bronchial asthma in childhood. During the follow-up period, all the children had been regularly screened for extensive helminthic infestations (enterobiasis, ascaridiasis) and lambliasis. The patients were grouped by invasion in the following way: Group 1 - children who had never had a single episode of invasion during the follow-up period (48 patients), and Group 2 - children who had been diagnosed with and successfully treated for enterobiasis, ascaridiasis, or lambliasis (42 patients). The catamnestic analysis also assessed the incidence of skin and respiratory manifestations of allergy, and compared the frequency of remission that lasted less than 3 years, from 3 to 6 years, from 6 to 12 years, and for over 12 years.

Results Among all the children with invasions, the first episode of bronchial obstruction was the onset of allergic pathology in half of the cases; at the same time, the same was true only for $15 \%$ of the children without invasions. Despite therapy against bronchial asthma, a sustained long-lasting remission of respiratory symptoms was absent in $44 \pm 6 \%$ children of Group 1 and in $43 \pm 7 \%(\mathrm{p}<0,05)$ children of Group 2 at the age of 18 . Drug-free remission of bronchial asthma and the absence of any other symptoms of allergy for over 12 years were found in 2 children without invasions $(7 \%)$ and 5 times more frequently $(n=9 ; 37 \%)$ in children with invasions.

Conclusions The collected follow-up data suggest that respiratory symptoms developing alongside immunology shifts triggered by a parasitic invasion are, as a rule, considered as onset of bronchial asthma. However, if the condition was diagnosed early in preschool children, and adequate antiparasitic therapy was given, about $15 \%$ of the patients had no clinical symptoms of allergic inflammation later in life. 